Foreword

For reprint orders, please contact: reprints@futuremedicine.com

\section{Biomarker discovery using dry-lab technologies and high-throughput screening}

\author{
"This special issue of Biomarkers in Medicine focuses on the \\ discovery of biomarkers or biomarker panels using dry-lab, \\ computational technologies, which provide rapid, systemic \\ calculation, comprehensive combinatory network information \\ and then could accelerate the efficiency with which biomarkers \\ can be applied to clinical practice."
}

First draft submitted: 22 April 2016; Accepted for publication: 22 April 2016; Published online: 6 June 2016

Keywords: bioinformatics $\bullet$ computational biology $\bullet$ high-throughput $\bullet$ miRNA $\bullet$ network - simple sequence repeat $\bullet$ wet and dry lab

Disease biomarkers can be discovered using two distinct approaches: wet and dry lab. Biomarker identification using wet-lab experiments involves the assessment of phenotypes, mechanisms or causes of disease. With respect to source material, blood is the easiest sample with which to isolate the desired analyte for subsequent biomarker detection. Identification of serum protein biomarkers in the secretome is practical for clinical or biomedical studies; indeed, biomarkers for tumor development and microbial infection as well as inflammatory, coronary, renal and other diseases (except brain diseases) are usually identified using serum or plasma samples [1-3]. For neurodegenerative diseases or mental disorders, however, serum or plasma protein markers are always challenging. Cerebrospinal fluid is a rational biopsy for detecting brain-disease biomarkers [4]. Moreover, high-throughput technology provides a powerful tool with which to facilitate biomarker identification regardless of whether the sample is plasma, serum or cerebrospinal fluid. In addition, computational methodology can be employed to analyze the 'big data' generated by high-throughput techniques in combination with biological networks, regulatory pathways and/or protein-protein interaction datasets [5-8]. This special issue of Biomarkers in Medicine focuses on the discovery of biomarkers or biomarker panels using dry-lab, computational technologies, which provide rapid, systemic calculation, comprehensive combinatory network information and then could accelerate the efficiency with which biomarkers can be applied to clinical practice.

We invited an Editorial contributed by T-W Pai (National Taiwan Ocean University, Taiwan) who discussed simple sequence repeats (SSRs), which are widely distributed in the genome of various species. Comparison between healthy control and disease subjects facilitates the discovery of genomic SSR variations, identified based on information from next-generation sequencing technology and statistical/predictive analyses with computational approaches. T-W Pai mentions that "SSRs that are well conserved across species might convey important messages for gene regulation in the cell, and polymorphic SSR markers found in human-unique genes can be applied as distinctive features for identifying different individuals." The Editorial presents analytic methodologies and databases for the application of genetic SSR markers to issues in the life sciences, agriculture and biomedicine [9].

\section{Biomarkers \\ in Medicine}
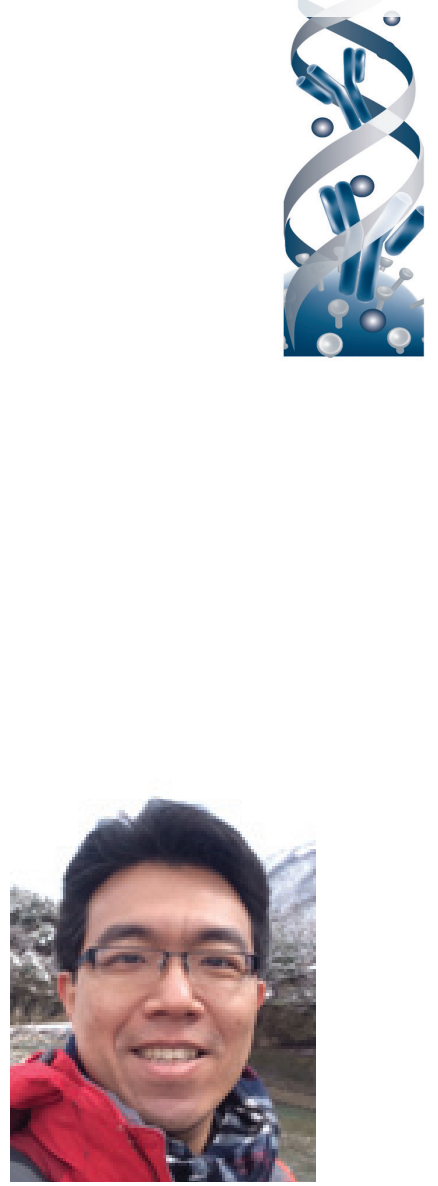

Hao-Teng Chang

Graduate Institute of Basic Medical Science, China Medical University, Taichung City, Taiwan

and

Department of Computer Science \& Information Engineering, Asia University, Taichung City, Taiwan Tel.: +886422052121 ext. 7721 htchang@mail.cmu.edu.tw 
This special issue also contains five review articles, including three full-length reviews, one systematic meta-analysis, and one special report. Z-P Liu (Shandong University, China) contributes an in-depth review that introduces the most common computational methods for identifying biomarkers of complex diseases using high-throughput technologies. He also summarizes representative network-based methodologies to highlight the importance of bioinformatics in biomarker discovery [10]. T Akutsu and colleagues (Kyoto University, Japan) also review relevant complex network-based approaches that have been used for biomarker discovery. These two reviews emphasize the significance of computational methodologies to assist the discovery of disease-related biomarkers [11]. J Zhang (Zhejiang University, China) reviews the potential of assessing circulating mRNAs for breast cancer diagnosis. His group reports a meta-analysis model with $88 \%$ sensitivity, $84 \%$ specificity and a 0.93 area under the curve value [12]. H-T Chang (China Medical University, Taiwan) reviews potential serum and bile markers for the diagnosis and prognosis of cholangiocarcinoma. In this regard, metalloproteases, serotonin, C-reactive peptide and bile acids are very promising for discriminating benign and malignant biliary conditions [13]. C-H Tang (China Medical University, Taiwan) reviews the potential for characteristics of the adipokine resisting as well as other adipokines as biomarkers for predicting angiogenesis and pathogenesis of rheumatoid arthritis [14]. These five reviews summarize the comprehensive knowledge on biomarkers for disease diagnosis/prognosis and for studies of disease mechanisms.

In addition, this issue contains four research articles. Y Wang (Chinese Academy of Sciences, China) reports a methodology to construct a combined-biomarker panel from multiple level datasets. Using a combination of serum assays, mass spectrometry and computational modeling, Wang's group identified a combined-biomarker panel based on analysis of 10 serum

\section{References}

1 Prieto DA, Johann DJ Jr, Wei BR et al. Mass spectrometry in cancer biomarker research: a case for immunodepletion of abundant blood-derived proteins from clinical tissue specimens. Biomark. Med. 8(2), 269-286 (2014).

2 Ohlendieck K. Proteomic identification of biomarkers of skeletal muscle disorders. Biomark. Med. 7(1), 169-186 (2013).

3 Di Girolamo F, Del Chierico F, Caenaro G, Lante I, Muraca M, Putignani L. Human serum proteome analysis: new source of markers in metabolic disorders. Biomark. Med. 6(6), 759-773 (2012). molecules and 6 mass spectra peaks, and this approach reached $87 \%$ accuracy. This methodology may help to create other cancer marker panels [15]. M Yoshida (Kobe University, Japan) employed metabolomics methodology to screen pancreatic cancer. Yoshida's group analyzed the metabolomes of 59 patients and 59 healthy controls and constructed a 2-phase screening model to distinguish pancreatic cancer from control samples. The model reached $84.1 \%$ sensitivity and 84.1\% specificity [16]. C-S Hwang (National TangMing University, Taiwan) identified single-nucleotide polymorphisms characteristic of amyotrophic lateral sclerosis in a Taiwanese Han population using a genome-wide association study. Among 54 relevant polymorphisms, several were significantly associated with neural-related Gene Ontology terms and related diseases. Two candidate loci were strongly associated with the disease [17]. Finally, X Xie (Zhejiang University in China) evaluated platelet distribution width in patients with chronic obstructive pulmonary diseases who also had a pulmonary embolism. In this retrospective study, platelet distribution width was found to be greater in these patients [18].

We thank all the contributors for their thorough review and reporting of the relevant clinical associations, predictive methods, genomic polymorphisms, serum marker panels and network-based methodologies for disease biomarker identification and discovery. We trust that this special issue will contribute to the improvement of biomarker studies.

\section{Financial \& competing interests disclosure}

The author has no relevant affiliations or financial involvement with any organization or entity with a financial interest in or financial conflict with the subject matter or materials discussed in the manuscript. This includes employment, consultancies, honoraria, stock ownership or options, expert testimony, grants or patents received or pending, or royalties.

No writing assistance was utilized in the production of this manuscript.

4 Vasic N, Connemann BJ, Wolf RC, Tumani H, Brettschneider J. Cerebrospinal fluid biomarker candidates of schizophrenia: where do we stand? Eur. Arch. Psychiatry Clin. Neurosci. 262(5), 375-391 (2012).

5 Wuolikainen A, Jonsson P, Ahnlund M et al. Multiplatform mass spectrometry analysis of the CSF and plasma metabolomes of rigorously matched amyotrophic lateral sclerosis, Parkinson's disease and control subjects. Mol. Biosyst. 12(4), 1287-1298 (2016).

6 Buoli M, Caldiroli A, Cumerlato Melter C, Serati M, de Nijs J, Altamura AC. Biological aspects and candidate biomarkers for psychotic bipolar disorder: a systematic review. Psychiatry Clin. Neurosci. 10, 26969211 (2016). 
$7 \quad$ Lv H, Wang Q, Wu S et al. Neonatal hypoxic ischemic encephalopathy-related biomarkers in serum and cerebrospinal fluid. Clin. Chim. Acta 23(450), 282-297 (2015).

8 O'Connor KC, Roy SM, Becker CH, Hafler DA, Kantor AB. Comprehensive phenotyping in multiple sclerosis: discovery based proteomics and the current understanding of putative biomarkers. Dis. Markers 22(4), 213-225 (2006).

9 Pai TW, Chen CM. SSR genetic markers in human genomes and their observable relationship to hereditary diseases. Biomark. Med. 10(6), 563-566(2016).

10 Liu ZP. Identifying network-based biomarkers of complex diseases from high-throughput data. Biomark. Med. 10(6), 633- 650 (2016).

11 Hayashida M, Akutsu T. Complex network-based approaches to biomarker discovery. Biomark. Med. 10(6), 613-619 (2016).

12 Xie S, Wang Y, Liu H. Diagnostic significance of circulating multiple microRNAs in breast cancer: a systematic review and meta-analysis. Biomark. Med. 10(6), 661-674 (2016).
13 Wang B, Chen L, Chang HT. Potential diagnostic and prognostic biomarkers for cholangiocarcinoma in serum and bile. Biomark. Med. 10(6), 613-619 (2016).

14 Su CM, Huang CY, Tang CH. Characteristics of resistin in rheumatoid arthritis angiogenesis. Biomark. Med. 10(6), 651-660 (2016).

15 Zou M, Zhang PJ, Chen L, Tian YP, Wang Y. Identifying joint biomarker panel from multiple level dataset by an optimization model. Biomark. Med. 10(6), 567-575 (2016).

16 Sakai A, Suzuki M1, Kobayashi T. Pancreatic cancer screening using a multiplatform human serum metabolomics system. Biomark. Med. 10(6), 577-586 (2016).

17 Chen CJ, Chen CM, Pai TW, Chang HT, Hwang CS. A genome-wide association study on amyotrophic lateral sclerosis (ALS) in the Taiwanese Han population. Biomark. Med. 10(6), 597-611 (2016).

18 Wang M, Zhang J, Ji Q. Evaluation of platelet distribution width in chronic obstructive pulmonary disease patients with pulmonary embolism. Biomark. Med. 10(6), 633-650 (2016). 